\title{
Partial reinforcement and latent inhibition effects on stimulus-outcome associations in flavor preference conditioning
}

\author{
Andrew R. Delamater
}

Published online: 8 April 2011

(C) Psychonomic Society, Inc. 2011

\begin{abstract}
Two experiments with thirsty rats explored the harmful effects of non-reinforced exposures to a flavor cue in the control by sensory-specific flavor-sucrose associations in a conditioned flavor preference paradigm. Experiment 1 demonstrated that rats learned to prefer a flavor cue that was consistently paired with sucrose over one that was paired with sucrose the same number of times but was also presented without sucrose on other occasions. However, rats for which sucrose was devalued following the conditioning phase preferred the partially reinforced flavor cue over the consistently reinforced flavor, suggesting that nonreinforcement weakened the ability of that flavor cue to evoke a specific representation of sucrose during the preference test. Experiment 2 demonstrated comparable effects of non-reinforcement in a latent inhibition procedure, although relatively more non-reinforced pre exposures to the flavor, in conjunction with fewer flavor-sucrose pairings, were required to see the effect. Together, the results suggest, as is often found with more traditional learning paradigms, that non-reinforcement of a flavor cue has deleterious effects on preference learning and/or performance.
\end{abstract}

Keywords Partial reinforcement - Latent inhibition .

Extinction - Flavor preference - Evaluative conditioning .

Configural learning

When neutral flavor or taste cues are paired with motivationally significant nutrients, a conditioned flavor preference is established for those cues when tested against

A. R. Delamater $(\bowtie)$

Psychology Department Brooklyn College-CUNY,

2900 Bedford Ave,

Brooklyn, NY 11210, USA

e-mail: andrewd@brooklyn.cuny.edu control cues that have previously been unpaired with the nutrient. This phenomenon is readily observed in both human and nonhuman subjects alike (see, e.g., Boakes 2005; Sclafani \& Nissenbaum, 1988). One of the intriguing aspects of the phenomenon is that learned flavor preferences are often reported to be highly resistant to extinction procedures (Capaldi, Myers, Campbell \& Sheffer, 1983; Drucker, Ackroff \& Sclafani 1994; Elizalde \& Sclafani, 1990; Harris, Shand, Carroll and Westbrook 2004). For instance, Harris et al. trained thirsty rats to associate an almond flavor with $4 \%$ sucrose by allowing rats to drink a mixture of the two on several occasions. When later presented with a choice between the almond flavor alone (without sucrose) and plain water, the rats preferred the almond solution much more so than did the unpaired controls. Strikingly, the rats maintained this preference for almond over 14 consecutive tests. In other words, although the almond flavor was paired with a relatively weak concentration of sucrose, the preference that was established was robust, showing no sensitivity to the fact that the two were no longer paired over what amounted to many extinction trials. Other authors have observed this result as well when using different conditioning and test procedures (e.g., Capaldi et al., 1983; Drucker et al., 1994; Elizalde \& Sclafani, 1990), and similar findings have also been reported with humans (e.g., Boakes, 2005; Stevenson, Boakes \& Wilson, 2000).

The phenomenon is so well established that it has prompted some to suggest that flavor preference learning represents a form of conditioning that distinguishes itself from most other laboratory-based learning paradigms. De Houwer, Thomas and Baeyens (2001), for instance, have suggested that flavor preference conditioning is a form of evaluative conditioning that differs from simple Pavlovian conditioning in this important respect. Similarly, Pearce 
(2002) offered a somewhat specialized configural-learning explanation for why conditioned flavor preferences fail to extinguish.

Complicating the analysis of flavor preference conditioning as a unique form of learning or one that obeys specialized configural-learning principles is the occasional finding in the literature that extinction treatments do sometimes have detectable effects in this learning paradigm. For example, Delamater (2007) reported that extinction reduced control by sensory-specific associations in a flavor preference paradigm under conditions in which preference between extinguished and non extinguished flavors was assessed. In one experiment, two flavor cues were first paired with the same nutrient (sucrose) on separate conditioning trials, and this was followed by an extinction phase in which only one of the flavors was presented without sucrose. Subsequently, sucrose was devalued in some subjects, but not in others, and, finally, all the subjects were given a choice between the two flavor cues (without sucrose). The results of this test revealed that non devalued rats preferred the non extinguished flavor but devalued rats preferred the extinguished flavor. The findings suggest that the extinguished flavor was less effective than the non extinguished flavor at evoking a representation of the specific sensory properties of sucrose (that were, themselves, devalued). In this way, both effects of extinction on the resulting preference could be accounted for (see also Harris et al., 2004).

Moreover, a recent study by Dwyer, Pincham, Thein and Harris (2009) provided evidence that is in direct conflict with the evaluative conditioning interpretation of flavor preference learning. The interpretation asserts that rats acquire a preference because the flavor cue undergoes a relatively permanent change in its hedonic evaluation as a result of being paired with a highly palatable nutrient. Along these same lines, Harris et al. (2004) suggested that their rats displayed a highly persistent flavor preference because the positive hedonic response that became conditioned to the flavor cue was highly resistant to extinction. However, in the Dwyer et al. study (2009), while rats displayed a preference for a flavor cue paired with $16 \%$ sucrose over one paired with $2 \%$ sucrose that was undiminished after several sets of test sessions, a lick cluster analysis revealed that differential lick clustering displayed to the two flavor cues did undergo extinction. Since higher lick clusters are often taken as a relatively direct measure of palatability, this experiment demonstrates that a learned flavor preference can be shown to persist in extinction despite reductions in cue palatability.

Finally, several investigators have also reported that conditioned acceptance of a flavor cue is relatively more sensitive to extinction procedures than conditioned preference appears to be (e.g., Drucker et al., 1994; Harris et al.,
2004; Higgins \& Rescorla, 2004). In these experiments, it has been observed that rats will drink more in a singlebottle test of a flavor cue that was previously paired with a nutrient than of one that was not. Furthermore, repeated non reinforced extinction tests commonly reveal in these studies that the difference between these two conditions in single-bottle intakes is diminished. In other words, extinction reduces the conditioned acceptance effect, even under conditions where a subsequent two-bottle test reveals little effect of extinction (e.g., Drucker et al., 1994).

The three sets of results described above are particularly problematic for any view that stresses the uniqueness of flavor preference learning on the basis of its failure to extinguish, because they suggest that extinction does, in fact, undermine control by at least some components of flavor preference learning. The results of Delamater (2007; see also Scarlet, Campese \& Delamater 2009) also point to the importance of using appropriate assessment procedures in detecting an extinction effect; for example, comparing non extinguished with extinguished stimuli seems to be particularly sensitive for detecting such effects. Nevertheless, although these results suggest that extinction effects can be obtained in this learning paradigm, it is not known how general are the effects of non-reinforcement upon learning or, at the very least, control by that learning. Two other procedures that involve separate non reinforcement of the cue include partial reinforcement and latent inhibition tasks. It has been widely observed in other conditioning paradigms that both of these tasks result in impairments in conditioned responding (e.g., Bouton \& Sunsay, 2003; Gottlieb, 2005; Lubow, 1989; Rescorla, 2007), but whether or not this also applies to learning in a conditioned flavor preference task has not been studied extensively (but see De la Casa, Marquez \& Lubow, 2009). The purpose of the present experiments, therefore, was to extend the generality of our extinction findings by determining whether separate non reinforced presentations of a flavor cue might impair control by sensory-specific flavor-nutrient associations in partial reinforcement (Experiment 1) and latent inhibition (Experiment 2) tasks.

The present studies are unique because of their focus on the formation of sensory-specific associations in flavor preference conditioning. It is widely recognized that flavor preference learning can result in a variety of associations that can influence intake. For instance, when a flavor cue is paired with a nutrient, such as sucrose, the cue can enter in to an association with the specific taste of sucrose (e.g., Delamater, Campese, LoLordo \& Sclafani, 2006; Dwyer, 2005; Harris et al., 2004), with the reinforcing post ingestive consequences of sucrose (e.g., Sclafani \& Nissenbaum, 1988), or with the positive hedonic response to sucrose (e.g., Dwyer, 2008; Harris et al., 2004). When one pairs an initially neutral flavor cue with the oral 
consumption of sucrose and then demonstrates that rats prefer this flavor cue to another that has not been so paired, this preference can be explained by the operation of any one of these flavor-nutrient associations. It becomes important, therefore, if we wish to determine the effects of various manipulations on "preference" learning, to actually use tasks that permit a specific assessment of one or the other of these different components of learning. Exactly this strategy has been adopted rather successfully by Sclafani and his colleagues with their use of a procedure (intragastric nutrient infusions) that makes possible a direct assessment of associations between flavor cues and the post ingestive consequences of nutrients (see, e.g., Sclafani \& Nissenbaum, 1988). The focus of the present experiments will be to assess the effects of non reinforcement on associations between the flavor cue and the specific sensory properties of the nutrient. As will be described below, this requires use of a highly specialized task that permits such an assessment.

\section{Experiment 1}

The present experiment was modeled after the betweengroup extinction study reported by Delamater (2007). That study is especially noteworthy because of its use of a reinforcer devaluation procedure (see Rozeboom, 1958) in order to gauge the associations formed between the flavor cue and the sensory-specific features of the nutrient unconditioned stimulus. As was noted in the general introduction, use of this technique allows for a more precise assessment of the nature of the learning established as a result of flavor-nutrient pairings than is commonly employed in the literature (see also Delamater et al., 2006; Dwyer, 2005; Harris et al., 2004; Scarlet et al., 2009). However, whereas in the earlier extinction study all non reinforced presentations of the flavor cue occurred after the conditioning phase, in the present experiment all non reinforced cue presentations occurred intermixed throughout the conditioning phase to form a partial reinforcement procedure. The experimental design appears in Fig. 1.

Each of two flavor cues (F1, F2) was paired with sucrose the same number of times, but one of these flavor cues (F1) was also presented a number of times without sucrose. In the next phase of the experiment, one subgroup of rats received a sucrose devaluation treatment, in which sucrose was presented by itself, and this was followed immediately by an injection of lithium chloride $(\mathrm{LiCl})$. A second subgroup of rats during this phase of the experiment received sucrose alone and $\mathrm{LiCl}$ on alternate days and, thus, served as a non devalued control group. In the test phase, all the subjects were given two-bottle tests involving a choice between F1and F2 without sucrose present.
Experiment 1: Partial Reinforcement

\begin{tabular}{l|l|l} 
F1+Sucr & Sucr-LiCl & F1 vs F2 \\
\cline { 2 - 3 } $2+$ Sucr & Sucr/LiCl &
\end{tabular}

Experiment 2: Latent Inhibition

\begin{tabular}{l|l|l|l} 
F1- & F1+Sucr & Sucr-LiCl & F1 vs F2 \\
\cline { 3 - 4 } & F2+Sucr & Sucr/LiCl &
\end{tabular}

Fig. 1 Experimental designs used in Experiments 1 and 2. F1 and F2 refer to different flavor stimuli (almond and banana, counterbalanced). Sucr refers to an $8 \%$ sucrose solution. $\mathrm{LiCl}$ refers to lithium chloride. See the text for additional details

On the assumption that non-reinforced presentations of a cue flavor reduces its ability to associatively activate a representation of the sensory properties of sucrose during the test, two important predictions follow. First, in non devalued control subjects, F2 should be preferred to F1, because the former should be more effective than the latter at evoking a representation of the highly valuable sensory properties (e.g., the palatable sweet taste) of the nutrient that support a preference. It seems possible, however, that the inclusion of non-reinforced flavor exposures might only weakly reduce the ability of the flavor cue in question to activate the representation of the sensory properties of sucrose. In that case, the ability to detect such an effect would be enhanced by the fact that one flavor cue that has been both reinforced and non-reinforced and a second one that has only been reinforced are being tested. These conditions should optimize the chances of detecting an effect of non-reinforcement (see also Delamater, 2007). Second, if non-reinforcement weakens the ability of the flavor cue to associatively activate a representation of the sensory properties of sucrose, devalued subjects should avoid F2 in the choice test. This prediction follows from the assumption that $\mathrm{F} 2$ will be more effective than $\mathrm{F} 1$ at evoking a sensory representation of the now devalued nutrient. There is clear evidence from the aversionconditioning literature that when an aversion has been established to sucrose, its sensory properties become aversive (see, e.g., Berridge, Grill \& Norgren, 1981). If F2 more strongly activates the devalued and now aversive sensory representation of sucrose than does $\mathrm{F} 1$, it follows that given a choice between the two flavors, F1 would be the better of two evils, and rats should prefer this solution if they are thirsty. The present study examined these ideas.

It may be appreciated that the predictions noted above are derived by considering the sole contribution of sensoryspecific learning processes to the expression of learned flavor preferences. The situation becomes more complex if one considers the possible roles played by the other components of flavor preference learning noted above 
(e.g., flavor-calorie or flavor-hedonic-response associations). This is so because these other types of association should, for the most part, be completely insensitive to a US devaluation treatment. This issue will be revisited in the General discussion section.

\section{Method}

Subjects Thirty-two Long-Evans male (12) and female (20) rats were run in the experiment. The rats were bred at Brooklyn College and were derived from rats acquired from Charles River labs. They were approximately 8 months old at the beginning of the experiment and were housed individually in a colony room that was on a 14:10-h light: dark cycle. The rats were maintained with home cage chow available ad lib throughout the experiment, but water was available during two 15-min drinking periods separated by $5.5 \mathrm{~h}$ each day. These drinking periods occurred during the light cycle, starting approximately $2 \mathrm{~h}$ after the lights came on in the colony room.

All the subjects had earlier participated in a Pavlovianto-instrumental transfer-of-control experiment in which they were exposed to short-duration auditory and visual cues paired with liquid sucrose $(0.1-\mathrm{ml}$ droplets of a $20 \%$ solution) or food pellets $(45 \mathrm{mg})$ and in which they were trained to make lever press and chain pull instrumental responses for these reinforcements under variable-interval schedules while maintained under food restriction. Their assignments to the different conditions of the present experiment were orthogonal to their earlier history, and in between experiments, the rats were maintained under ad lib water and food conditions for a couple of months.

Apparatus and solutions The experiment was performed in the rats' home cages in the colony room, starting approximately $2 \mathrm{~h}$ after the lights were turned on. These cages were of standard dimensions $(24 \times 18 \times 17.5 \mathrm{~cm})$ and were made of wire mesh with solid stainless steel sidewalls. The various solutions were presented in 50-ml centrifuge tubes attached to the outside of the cage, with their metal spouts protruding approximately $4 \mathrm{~cm}$ into the front of the cage. Imitation almond and banana extracts (McCormick brand) were mixed in solution (1\%) with tap water and served as conditioned stimuli (CSs) in this experiment. The unconditioned stimulus (US) was an $8 \%$ sucrose solution, and this was mixed into solution with the appropriate extract. During the devaluation phase, $\mathrm{LiCl}(0.3$ Molar, $1 \%$ body weight) was administered through an intraperitoneal (i.p.) injection.

Procedure Initially, the rats were given several days to accommodate to the drinking schedule. They were permit- ted to drink tap water from the centrifuge tubes for $15 \mathrm{~min}$ twice a day, at 10:30 a.m. and 4:00 p.m. Following this, the conditioning phase began.

The conditioning phase was run in five 4-session blocks. Because there were two 15-mindrinking periods in each day, this meant that each 4-session block occurred over 2 days. In each block, both flavor cues were presented (irregularly and counterbalanced for time of day), once in solution with sucrose $(\mathrm{F} 1+, \mathrm{F} 2+)$, and one of the flavor cues was designated as the non reinforced cue and was presented alone twice (F1). Thus, there was a total of five pairings of each flavor cue with sucrose, plus another ten presentations of one of these flavor cues alone. For half of the subjects, the almond flavor was designated as the partially reinforced flavor (F1), and for the remaining animals, banana was so designated.

The sucrose devaluation phase began on the next day. It was run in three 2-day cycles. On the first day of each cycle, sucrose was presented for $15 \mathrm{~min}$ in one bottle during the a.m. session. Immediately after this session, half of the subjects (Gp Devalued) were taken to an adjacent room, were injected i.p. with $\mathrm{LiCl}$, and then were returned to their home cages. Plain water was presented for $15 \mathrm{~min}$ during the p.m. session. On the second day of each cycle, all the subjects consumed water during both the a.m. and p.m. sessions. The rats not injected on the previous day (Gp Non devalued) were given an i.p. injection of $\mathrm{LiCl}$ following the a. $\mathrm{m}$. water session on this day.

The test phase began on the day following the final devaluation cycle. All tests were conducted during the a.m. session, and subjects were given water to drink during the p.m. session. There were two test sessions, and in each, the rats were given a choice between almond and banana flavors for $15 \mathrm{~min}$. The position on the cages of these two solutions was counterbalanced across rats on each day, as well as within rats a cross days. Each test was run as follows. One of the solutions was presented until the rat sampled it (approximately $2 \mathrm{~s}$ ) before it was withdrawn and replaced by the other flavor until the rat sampled it (for the same time) before it was withdrawn, and then the two tubes were reinserted into the cage separated by approximately $3 \mathrm{~cm}$. Both tubes were withdrawn 15 min later.

Statistical analysis Here and throughout, analysis of variance (ANOVA) techniques were used to analyze the intake data. Where appropriate, post hoc contrasts were conducted using the methods recommended by Rodger (1974) to ensure a type I error rate of 0.05 . Note that use of Rodger's techniques provides for greater power in detecting true effects, because these techniques do not suffer a reduction in power as numerator degrees of freedom increase (as more typical procedures do). This makes these techniques especially sensitive for detecting interactive effects when they exist. The bottles were weighed before and after the 
session to arrive at an amount-consumed score measured to the nearest $0.1 \mathrm{~g}$.

\section{Results}

Although intakes were generally lower for female rats than for males, all of the data patterns reported in these experiments were seen in both of these subgroups. For the purposes of all statistical analyses, therefore, the data have been collapsed across the sex factor. Over the course of the acquisition phase, intakes gradually increased for all three solutions: $\mathrm{F} 1, \mathrm{~F} 1+\mathrm{S}$, and $\mathrm{F} 2+\mathrm{S}$. In addition, intake of the two sucrose solutions $(\mathrm{F} 1+\mathrm{S}, \mathrm{F} 2+\mathrm{S})$ was greater than that of F1 alone. In block 5 of training, the mean intakes for F1, $\mathrm{F} 1+\mathrm{S}$, and $\mathrm{F} 2+\mathrm{S}$ were $10.2,19.1$, and $19.7 \mathrm{~g}$, respectively. A one-way ANOVA applied to these data revealed a significant main effect of flavor, $F(2,62)=107.09$. Subsequent post hoc tests revealed that the two flavor + sucrose solutions did not differ from one another but that both were consumed more than $\mathrm{F} 1$ alone, $F(2,62)=107.28$.

Sucrose intakes during the devaluation phase steadily decreased in Gp Devalued, but not in Gp Non devalued. Intakes in each of the three cycles were $21.8,8.5$, and $2.0 \mathrm{~g}$ for Gp Devalued rats, and they were 22.6, 18.7, and $19.2 \mathrm{~g}$ for $\mathrm{Gp}$ Non devalued rats. A cycle $\times$ devaluation ANOVA on these data revealed reliable main effects of devaluation, $F(1,30)=66.24$, and cycle, $F(2,60)=100.06$, as well as a reliable devaluation $\times$ cycle interaction, $F(2,60)=47.00$.

The primary data of interest came from the F1 versus F2 choice tests. The data were collapsed over the two test sessions (in order to form a complete position counterbalancing within subjects) and are presented in Fig. 2. For each group, flavor intakes are shown for the partially reinforced F1 and the continuously reinforced F2 flavors. As can be seen, Gp Non devalued subjects preferred the

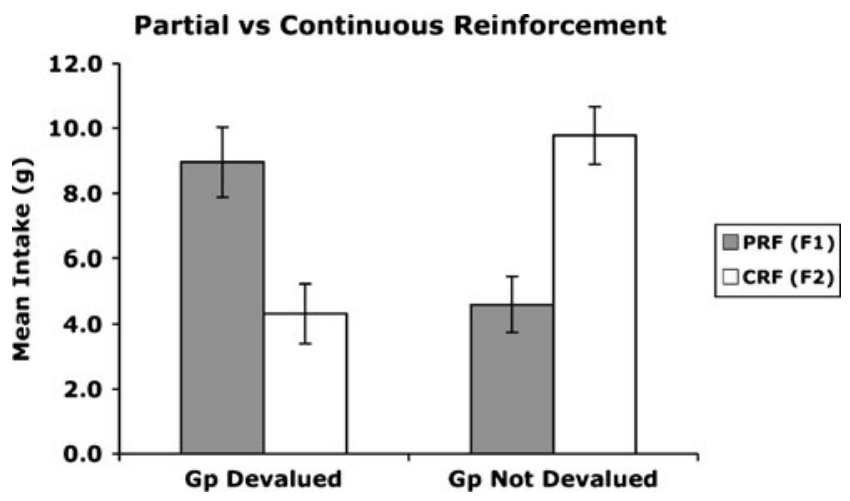

Fig. 2 Mean intakes for Group Devalued and Gp Non devalued subjects of the partially reinforced [PRF (F1)] and continuously reinforced $[\mathrm{CRF}(\mathrm{F} 2)]$ flavor cues during the choice test in Experiment 1 continuously reinforced F2 flavor, but Gp Devalued subjects preferred the partially reinforced F1 flavor.

These data were analyzed using a devaluation (devalued, non devalued) $\times$ flavor $(F 1$, F2) ANOVA that revealed a reliable devaluation $\times$ flavor interaction, $F(1,30)=15.84$. This analysis was followed up with a separate one-way ANOVA, using an appropriately pooled error term, performed across the flavor factor for each group. This confirmed that F2 was preferred to F1 in Gp Non devalued, $F(1,30)=8.83$, and that $\mathrm{F} 1$ was preferred to $\mathrm{F} 2$ in $\mathrm{Gp}$ Devalued, $F(1,30)=7.06$.

\section{Discussion}

The results from the present study confirm that non reinforcing a flavor cue that is otherwise paired with sucrose reduces the effective control by the sensory-specific flavor-sucrose association normally formed when a flavor cue is paired with a nutrient. As compared with a flavor cue that was equally paired with sucrose but lacked any additional non reinforced presentations, the continuously reinforced flavor was preferred over the partially reinforced flavor in non devalued control subjects. Gp Devalued subjects, in contrast, displayed a strong preference for the partially reinforced flavor cue. Both results parallel those reported by Delamater (2007) in showing that non reinforcement effectively reduces control by sensoryspecific associations in the same manner as in the present experiment, with the exception that, in that study, all non reinforced presentations occurred after, not intermixed with, training. The present results, therefore, add to the generality of those findings by showing similar reductions in control by sensory-specific flavor-nutrient associations brought about by nonreinforcement. Apparently, such nonreinforcements reduce the degree to which these flavor cues are able to activate a sensory-specific representation of sucrose at the time of test, as compared with a continuously reinforced flavor, and this is responsible for the pattern of results obtained here.

Although the data were not analyzed in this way, examination of the data in Fig. 2 suggests that the partially reinforced flavor, F1, was consumed more in Gp Devalued than in Gp Non devalued. At first blush, this result may appear anomalous, because an aversion had been conditioned to sucrose in Gp Devalued, and if one were to assume that the strength of the F1-sucrose association was equivalent in the two groups, Gp Non devalued might be expected to consume higher levels of F1. It should be noted, however, that F1 intakes were assessed in the context of a preference test involving a choice between F1 and F2. Therefore, this apparent between-group difference is very likely a consequence of the fact that the rats were thirsty going into the test and F2 was strongly aversive in Gp Devalued. This would have raised F1 intakes in that group. 
One concern regarding the present data is that non-naïve animals were used in the experiments reported here. In both experiments, rats that had earlier performed in a Pavlovianto-instrumental transfer task, where they had experience with both food pellet and $20 \%$ sucrose USs while food was restricted, were used. In the present study, flavor cues were used (rather than the auditory and visual cues used in the former task), paired with $8 \%$ sucrose, while rats were water restricted. In the earlier study, rats had experienced a highly concentrated sucrose solution in $0.05-\mathrm{ml}$ quantities, where as in the present study, they consumed an unlimited amount of a less concentrated sucrose solution from a bottle. It is not at all clear to what extent the rats generalized between these two solutions. Nevertheless, Delamater (2007) similarly used non-naïve rats to explore the effects of extinction on sensory-specific flavor-nutrient associative learning, and in one study, a within-group assessment was made with waterrestricted rats in which training occurred with two distinct USs. One of these was the same weaker concentration of sucrose as that used in the present experiments, and the other was Polycose, a nutrient with which they had no prior experience. The results of that study revealed no differences as a function of nutrient type. In other words, the effect of nonreinforced extinction trials was to equivalently impair control by sensory-specific associations with both sucrose and Polycose USs. Therefore, it is highly unlikely that the results of the present study are related to the fact that the rats had prior exposure to smaller quantities of a much more concentrated sucrose solution, while food was restricted.

\section{Experiment 2}

The present experiment explored the effects of non reinforcement on flavor preference conditioning, using a latent inhibition procedure. Figure 1 shows the experimental design. The experiment was similar to that used in Experiment 1, except that all non reinforced F1 presentations occurred prior to the conditioning phase in which $\mathrm{F} 1+$ sucrose and F2 + sucrose pairings were given. The effects of devaluing sucrose upon preference for F1 and F2 were then examined in several choice tests. In a pilot study, it was observed that when the same number of reinforced and nonreinforced trials was used as in Experiment 1, a basic latent inhibition effect in non-devalued animals did not occur, probably because too many conditioning trials overcame the effects of nonreinforced preexposures. Therefore, in this experiment, the number of preexposures was increased and the number of conditioning trials was decreased, in an effort to optimize our chances of detecting latent inhibition effects.

It may be noted that there is only one study in the literature that has demonstrated a latent inhibition effect in flavor preference learning (De la Casa et al., 2009). The present experimental design was quite similar to that used by De la Casa et al. but also differed in some important respects. First, whereas De la Casa et al. trained rats while they were under a food and water restriction regime, the animals in the present experiment (as in Experiment 1) were only water restricted. In addition, in the present experiment, following the conditioning phase, sucrose was paired with $\mathrm{LiCl}$ in one group of rats but was unpaired with $\mathrm{LiCl}$ in another group of rats. This is a crucial difference in our experimental design, because, as was noted above, this feature allowed us to directly examine the status of sensoryspecific flavor-sucrose associations. The De la Casa et al. study demonstrated that rats preferred a flavor cue that was paired with sucrose over another flavor cue that was paired with sucrose but had also received nonreinforced preexposures. This result suggests that preference learning is sensitive to a latent inhibition procedure, but it does not allow us to determine what aspect of the learning was sensitive to this procedure. The present design allowed us to focus on the sensory-specific flavor-sucrose association and determine whether this would be in some way impaired by a preexposure manipulation.

\section{Method}

Subjects Subjects were 18 male and 14 female Long-Evans rats bred at Brooklyn College and derived from rats acquired from Charles River labs. Their age at the beginning of the experiment was approximately 8 months. The rats were housed and maintained as in Experiment 1 . The rats had earlier participated in a Pavlovian-toinstrumental transfer-of-control experiment. Their assignments to the different counterbalancing conditions in the present experiment were orthogonal to their earlier history. The rats were maintained as in Experiment 1.

Apparatus and solutions The same apparatus and solutions were used as in Experiment 1.

Procedure The procedures were the same as those in Experiment 1, with a few exceptions noted below. Over the first 8 days, the F1 flavor was presented during both a. $\mathrm{m}$. and p.m. sessions for a total of 16 nonreinforced preexposures to F1. Over the next 2 days, the F1 + sucrose and F2 + sucrose mixtures were each presented once per day. Whether these were presented in the a.m. or the p.m. session was counterbalanced across animals and across days. The 6-day devaluation phase treatment began following the conditioning phase, and testing commenced on the day following devaluation training. In this experiment, there were a total of four test sessions (rather than only two, 
as in the previous experiment). Following the first two F1 versus F2 flavor test sessions, all the rats were given a sucrose versus water choice test to assess the degree of a sucrose aversion. This was followed by two more F1 versus F2 test sessions beginning on the next day.

\section{Results}

Intake of F1 was relatively constant across the pre exposure phase (mean intakes across days were 10.7, 11.5, 12.1, 11.1, $13.7,10.8,10.7$, and $12.0 \mathrm{~g}$ ). During the conditioning phase, intake of the F1 + sucrose mixture was higher than the novel F2 + sucrose mixture on day 1 (means were 17.7 and 8.0, respectively). However, this difference disappeared on day 2 , where intakes of F1 + sucrose and F2 + sucrose were 14.9 and $13.6 \mathrm{~g}$, respectively. A one-way ANOVA confirmed that $\mathrm{F} 1+$ sucrose, F2 + sucrose, and F1-alone intakes differed, $F(2,62)=3.91$. Subsequent post hoc tests (Rodger, 1974) confirmed that the two flavor+sucrose solutions were consumed more on the final day of training than F1 alone was consumed by the end of preexposure, $F(2,62)=3.39$.

As in the previous experiments, sucrose intakes during the devaluation phase steadily decreased in Gp Devalued, but much less so in Gp Non devalued. Sucrose intakes in each of the three cycles were 20.9, 4.1, and $1.3 \mathrm{~g}$ for $\mathrm{Gp}$ Devalued rats, and they were 19.9, 16.6, and $14.6 \mathrm{~g}$ for $\mathrm{Gp}$ Non devalued rats. A cycle $\times$ devaluation ANOVA on these data revealed reliable main effects of devaluation, $F(1,30)=36.29$, and cycle, $F(2,60)=139.08$, as well as a reliable devaluation $\times$ cycle interaction, $F(2,60)=51.07$.

The groups also differed in their sucrose intakes during the sucrose versus water two-bottle test that occurred between the two sets of flavor tests. Sucrose was strongly preferred to water in Gp Non devalued (mean intakes of sucrose and water were 10.8 and $3.1 \mathrm{~g}$, respectively), but it was strongly avoided in Gp Devalued (mean intakes of sucrose and water were 1.6 and $10.2 \mathrm{~g}$, respectively). An ANOVA performed on these data revealed a significant solution $\times$ group interaction, $F(1,30)=43.66$.

The primary data of interest came from the $\mathrm{F} 1$ versus F2 choice tests. The data from the first and second sets of test sessions (before and after the sucrose vs. water tests, respectively) are presented in Fig. 3. For each group, flavor intakes are shown for the preexposed F1 and nonpreexposed F2 flavors. Gp Devalued subjects preferred the preexposed F1 flavor over the nonpreexposed F2 flavor, but Gp Non devalued subjects preferred the nonpreexposed F2 flavor over the preexposed F1 flavor. These effects appeared more prominently in the second set of test sessions.

Because statistical power is increased with increasing numerator and denominator degrees of freedom using
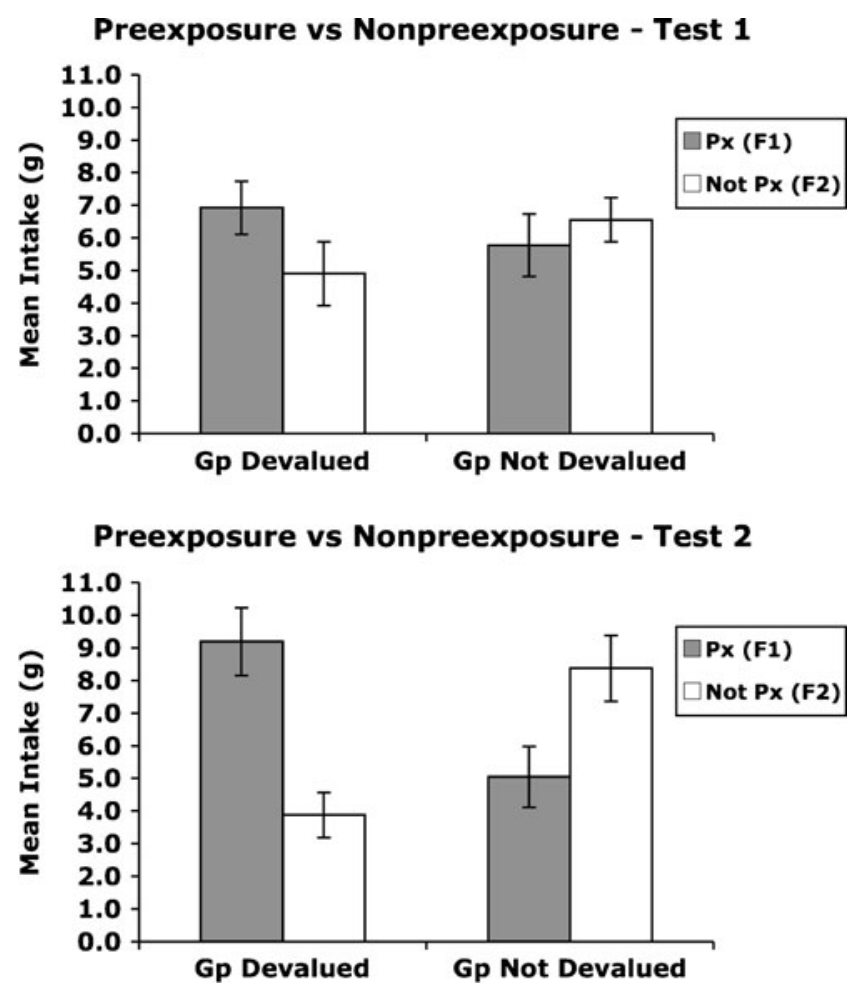

Fig. 3 Mean intakes for Group Devalued and Gp Non devalued subjects of the pre exposed $[\mathrm{Px}(\mathrm{F} 1)]$ and not pre exposed [Not $\mathrm{Px}(\mathrm{F} 2)]$ flavor cues during choice test 1 (top) and choice test 2 (bottom) in Experiment 2

Rodger's approach (1974), the data were analyzed by considering the test and flavor factors as one factor (test: flavor) with four levels (T1:F1,T1:F2, T2:F1, T2:F2). This combined factor was then examined against the group factor in an ANOVA. This analysis revealed a reliable group $\times$ test: flavor interaction, $F(3,90)=7.20$. This interaction was followed up with separate one-way repeated measures ANOVAs performed on each group, using a pooled error term. This analysis revealed differences in intakes across the conditions both for $\mathrm{Gp}$ Devalued, $F(3,90)=5.79$, and for Gp Non devalued, $F(3,90)=2.13$. Subsequent post hoc tests (Rodger, 1974) revealed that Gp Non devalued subjects preferred F2 over F1 in the second set of tests, $F(3,90)=1.93$, but that $\mathrm{Gp}$ Devalued subjects preferred F1 over F2 in the second set of tests, $F(3,90)=4.94$. Although the same general pattern of data was observed in the first set of tests, neither group displayed significant differences in those tests.

\section{Discussion}

The results of Experiment 2 suggest that nonreinforcement in a latent inhibition experiment also results in attenuated control by sensory-specific associations estab- 
lished during flavor-nutrient conditioning. It was observed that subjects preferred a nonpreexposed flavor over one that had been preexposed if the associated nutrient, sucrose, was valued at the time of test. This replicates the basic latent inhibition effect that De la Casa et al. (2009) observed in a flavor preference learning paradigm. However, our data also demonstrated that the reverse was true if sucrose had been devalued. It is these results, together, that strongly point to the fact that our preexposure manipulation in some fashion undermined control by the sensory-specific flavor-sucrose association acquired during the training phase.

It is of some interest that the latent inhibition effects reported here were more noticeably observed only after subjects were given a sucrose versus water test. It seems possible that this separate sucrose test could have contributed to the results. For instance, if during the nutrient test the devalued nutrient evoked a representation of its associated flavor cue, as well as a conditioned nausea response, then perhaps such dual activation provided an opportunity for the flavor cue to associate with illness. Such an effect would not be unprecedented. For instance, Dwyer (1999) demonstrated that an almond aversion was established when a context-evoked representation of almond was paired with LiCl-induced nausea. This result is consistent with our claim, if one assumes that after it has been associated with $\mathrm{LiCl}$, sucrose evokes an incipient nausea reaction not unlike that produced by $\mathrm{LiCl}$ itself. In this same report, however, Dwyer (1999) also failed to observe that rats would learn an almond aversion if a context-evoked representation of almond was paired with consumption of another flavor cue (i.e., peppermint) that had earlier been paired with $\mathrm{LiCl}$. The latter result seems inconsistent with the claim put forth here. However, it is important to note that the present claim is that sucrose might evoke a representation of its associated flavor cue, as well as an incipient nausea response. In Dwyer's (1999) experiment, a representation of almond was evoked by a contextual cue - a stimulus that was quite different from the event presumed to induce an incipient nausea reaction (i.e., peppermint). Thus, the procedures used here may have especially encouraged animals to couple the evoked representation of F1 with the incipient nausea reaction evoked by sucrose, because every moment that sucrose was sampled, it would concurrently evoke a representation of the flavor cue, as well as a nausea reaction. This is not the case when different stimuli come to evoke the two events to be associated in this manner.

These ideas notwithstanding, there is reason to be cautious in interpreting the present findings in this way. This study was not designed to specifically examine the effects of sucrose aversion tests on preference for the flavor cues, since all the subjects were tested in the same manner. Such an examination would be required before firmer conclusions can be reached concerning the role of these tests (and any presumed learning mechanisms) in flavor preferences.

\section{General discussion}

The present experiments examined the role of nonreinforcement in control by sensory-specific flavor-sucrose associations in a flavor preference learning paradigm using partial reinforcement and latent inhibition procedures. The results were consistent in showing that nonreinforced presentations of a flavor cue in both of these procedures impaired learning or, at the very least, the expression of that learning. In particular, these experiments demonstrated that after sucrose was devalued, subjects avoided a flavor cue that had been consistently paired with sucrose, relative to a second flavor cue that had been paired with sucrose the same number of times but that had also been presented without sucrose. In contrast, in subjects for whom sucrose was not devalued, a consistently reinforced flavor cue was preferred over a flavor cue that was either partially reinforced or latently inhibited.

These findings could most readily be understood if it were assumed that pairing a flavor cue with sucrose results in the formation of an association between the flavor cue and some specific sensory quality of sucrose (e.g., its sweet taste) (see also, Delamater et al., 2006; Dwyer 2005; Harris et al., 2004). If it were further assumed that nonreinforcement of the flavor cue impairs the ability of this cue to associatively activate a representation of the sensory qualities of sucrose, such a cue should be preferred to a consistently reinforced cue if sucrose was devalued but should be avoided if sucrose was not devalued. Exactly this logic was used by Delamater (2007) in showing comparable effects with a flavor cue that underwent conditioning and then extinction. The results, therefore, establish some generality to the claim that nonreinforcement impairs control by associative relations learned in a flavor preference task.

One noteworthy aspect of the present findings, together with the earlier extinction results, is that the effects of nonreinforcement were assessed by offering subjects a choice between two flavor cues, one of which was reinforced consistently and one of which was not. This procedure should be especially sensitive for detecting effects of nonreinforcement, because it arranges for an assessment of the relative strengths of control by flavornutrient associations when those strengths should be comparable in the absence of nonreinforcement. Other test procedures may fail to detect an effect of nonreinforcement, not because effective control by underlying associations is unimpaired, but because the testing method is less sensitive for detecting associative impairments. 
The conclusion that flavor preference learning reflects the operation of a unique form of learning (e.g., De Houwer et al., 2001) or some specialized configural process (e.g., Pearce, 2002) has been partly based on claims that learned flavor preferences are insensitive to extinction treatments. Delamater (2007) demonstrated that this need not be the case, and the present experiments confirm that other methods involving nonreinforcement of the flavor cue have similar deleterious effects on the underlying association between the flavor and the rather specific sensory qualities of the nutrient. Thus, the data argue against any strong claims of uniqueness in this paradigm.

It might be noted, however, that while the present results are in general agreement with a variety of findings from "standard" learning paradigms that nonreinforcement hinders conditioned responding (see, e.g., Gottlieb, 2005), there are relatively few examinations of the effects of non reinforcement on control by highly specific stimulusoutcome associations. For instance, Rescorla (1996) used a reward devaluation task in assessing the status of specific stimulus-outcome associations following extinction in a magazine approach paradigm. He failed to find evidence of any associative loss brought about by extinction. Delamater (1996) also failed to find any loss of control by specific stimulus-outcome associations following extinction in a magazine approach paradigm in which the measure of control was specific Pavlovian-to-instrumental transfer. Thus, while current theories of learning (e.g., Gallistel \& Gibbon, 2000; Pearce, 1987, 1994; Rescorla \& Wagner, 1972; Wagner, 2008) suggest that nonreinforcement should attenuate associative control, in some fashion, with rather precise measures of associative learning, the clearest evidence supporting this basic claim seems to come, ironically enough, from flavor preference learning studies. The findings reported here suggest that similar harmful effects of nonreinforcement on specific stimulus-outcome associations should be observable, at least under some circumstances, in other, more traditional paradigms as well.

Although the present findings, together with those of Delamater (2007), suggest that nonreinforcement of a cue reduces its associative control, these data do not allow for a determination of the specific manner in which associative control is reduced by nonreinforcement. For instance, one mechanism is that nonreinforcement results in reductions in associative strength (e.g., Rescorla \& Wagner, 1972), and this could be responsible for reductions in conditioned responding or, in the present circumstances, control by specific flavor-sucrose associations. Another mechanism is that nonreinforcement induces a new form of learning - for example, conditioned inhibition - that temporarily masks the expression of prior associative learning (see, e.g., Bouton, 2004). From the present data, it is not clear which, if either, of these mechanisms is responsible for our results.
Moreover, it is difficult to conclude from the present set of findings whether there are multiple mechanisms affecting the manner in which nonreinforcement reduces associative control in extinction, partial reinforcement, and latent inhibition procedures. However, other data are relevant to these issues.

It is interesting to compare the effects of non reinforcement in extinction and partial reinforcement procedures. Some previous research supports the view that similar processes are at work in both procedures, but other research points to important differences as well. Rescorla (1997, 2004a, 2004b) demonstrated spontaneous recovery of conditioned magazine approach responses to stimuli that had undergone extinction, and Rescorla (2007) demonstrated spontaneous recovery following training with a partial reinforcement procedure. In both cases, it has been assumed that nonreinforcement results in the superimposition of an inhibitory learning process upon an excitatory learning process to reduce over all levels of responding. If this inhibitory learning process loses its effectiveness over time more rapidly than the excitatory process, it follows that spontaneous recovery will occur in both circumstances.

On the other hand, Bouton and King (1986) presented data suggesting that nonreinforcement in partial reinforcement and extinction tasks function differently. In their experiment, reinstatement of conditioned fear responses was explored for conditioned and then extinguished stimuli and also for partially reinforced stimuli, but these authors observed context-dependent reinstatement of conditioned fear only for the former, but not the latter, type of cue. These authors suggested that only truly "ambiguous" stimuli would be affected by manipulations involving conditional control by context (such as reinstatement). The important implication here is that although both extinction and partial reinforcement procedures entail nonreinforcement and, presumably some form of inhibitory learning, only the extinction procedure results in a truly ambiguous stimulus capable of being modulated by conditional contextual control mechanisms. Taken together with the spontaneous recovery results of Rescorla (2007), the data, overall, suggest that nonreinforcement in extinction and partial reinforcement procedures may involve similar but different mechanisms.

It is also instructive to consider comparisons between extinction and latent inhibition procedures. According to some views, these two phenomena may both reflect the operation of a similar set of conditional-learning mechanisms (e.g., Bouton, 1991, 1993; but see Bouton \& King, 1986). In essence, whether or not there are separate memories for reinforcement and nonreinforcement that control performance in any given situation will depend critically on contextual factors. Consistent with this general approach, Leung and Westbrook (2010) have recently 
reported that in a fear-conditioning preparation, under some conditions, latent inhibition and extinction can have additive effects in determining the amount of spontaneous recovery, a result that suggests some commonality between the two processes. On the other hand, other research has called into question the view that nonreinforcement entails inhibitory learning in a latent inhibition procedure (e.g., Halgren, 1974; Rescorla, 1971). This has led authors to propose different mechanisms for extinction and latent inhibition phenomena (e.g., Pearce \& Hall, 1980; Wagner, 1978). Delamater, Campese and Westbrook (2009) recently provided evidence to support this view. They demonstrated that the contextspecific control over extinguished behavior in a renewal paradigm depends on a completely functioning GABAergic neurotransmitter system, whereas the context-specific expression of latent inhibition does not. In this manner, different neurotransmitter substrates may be involved in the contextual modulation of latent inhibition and extinction.

It is far from clear from all of the considerations above whether nonreinforcement works in similar or different ways in procedures such as latent inhibition, partial reinforcement, and extinction that differ only in the placement of nonreinforced trials relative to reinforced ones. Part of the problem is that systematic comparisons between these phenomena within the same learning paradigm have not been extensively examined. The present experiments demonstrate, at the very least, that effects of nonreinforcement can be obtained in partial reinforcement and latent inhibition procedures that are similar to those seen in extinction (Delamater 2007) within the same learning paradigm - flavor preference conditioning. Whether or not these similar findings imply similar underlying psychological and neural mechanisms in these different situations will have to await further research.

In the present article, I have emphasized the assessment of nonreinforcement for one aspect of flavor preference learning - namely, the association between the flavor CS and some very specific sensory property of the nutrient with which it is paired. However, one might question the degree to which such associations play a role in the expression of learned preferences more generally. Others have emphasized the fact that flavor cues can also associate with the post-ingestive consequences of nutrient consumption-for example, their calories or the reinforcement signal generated by processing those calories (see, e.g., Sclafani \& Nissenbaum, 1988), as well as, possibly, the positive hedonic responses to highly palatable nutrients (see, e.g., Dwyer, 2008). The present experiments were not designed to address questions concerning the relative contributions of these different types of associations to the expression of preferences more generally. However, it may be noted that in order for a US devaluation effect to be manifest in preference learning at all demands that the flavor cue had associated with some specific sensory characteristic of the nutrient. This requires, then, that the present data could not be explained without appeal to such a construct. This means that any associations that might form between the flavor cue and the hedonic response to sucrose could not in and of itself explain the present findings, for the simple reason that such associations do not encode the sensory qualities of sucrose that have been devalued.

It seems possible that post-ingestive signals may be sensory specific, and so there could be a basis for the flavors to associate with such signals. In order for this sort of analysis to apply to the US devaluation findings here, it would have to additionally be assumed that the devaluation effect was mediated by devaluation of those post-ingestive sensory-specific signals during sucrose aversion training. In pilot experiments, an attempt was made to directly condition an aversion to such signals by intragastrically administering a nutrient just prior to a $\mathrm{LiCl}$ injection. Although there exist some published data to suggest that such training results in decreased intake of that nutrient in a subsequent intake test (see Tracy \& Davidson, 2006; Tracy, Phillips, Chi, Powley $\&$ Davidson 2004), this result could not be obtained in our lab.

There is additional reason to be cautious about the role of post-ingestive processes in the present paradigm. Delamater (2007) examined the effects of extinction on flavor preferences in animals trained and tested thirsty in one experiment and hungry in another. The results suggested that post-ingestive processes contribute to the expression of a preference when animals are hungry but play little role when animals are thirsty. In Experiments 2 and 4 in that article, rats were trained to separately associate two flavors with sucrose and two other flavors with Polycose. Then one flavor associated with each nutrient was extinguished. Following this, one of the nutrients was associated with $\mathrm{LiCl}$, and, finally, the rats were given choice tests pitting the two cues (one extinguished and one not) associated with the same nutrient against one another. Rats were trained and tested while thirsty in one experiment and while hungry in another. The results were quite different in these two circumstances. In particular, when rats were thirsty throughout, the same pattern of results was obtained as that reported here. However, animals trained and tested while hungry strongly preferred the nonextinguished flavor over the extinguished flavor when the associated nutrient was valued, but they displayed the same preference in a weaker form when the associated nutrient was devalued. This more complex pattern of findings was interpreted to mean that thirsty rats' preferences were primarily controlled by sensory-specific flavor-nutrient associations but that hungry rats' preferences were controlled by both sensory-specific associations and more general flavor-calorie associations (Delamater, 2007). The 
puzzling result was why hungry rats still preferred (albeit to a weaker degree) the non-extinguished flavor cue when the associated nutrient had been devalued. If the preference in these rats was exclusively controlled by a general flavorcalorie association, which was partially undermined by extinction, rats should continue to prefer the nonextinguished flavor. However, even these rats were sensitive to the devaluation treatment, although their preference was not reversed, and this suggests that the sensory-specific flavor-nutrient association also contributed. The reason why the thirsty rats displayed a reversed preference (in favor of the extinguished cue) when the associated nutrient was devalued is most readily interpreted as meaning that the general flavor-calorie association contributed very little to their preference, leaving control to be dominated by the sensory-specific flavor-nutrient association.

In summary, the present data add to the growing body of evidence showing that rather specific flavor-nutrient associations are learned in flavor preference conditioning tasks (e.g., Delamater, 2007; Delamater et al., 2006; Dwyer 2005; Harris et al., 2004; Scarlet et al., 2009). Moreover, they support the view that control by such associations is impaired by procedures that involve nonreinforcement. As such, these findings are more consistent with traditional views of associative learning and call into question those views that regard flavor preference learning as highly specialized or unique (e.g., De Houwer et al., 2001; Pearce 2002).

Acknowledgements This research was supported by National Institute of Mental Health (065947) and Professional Staff Congress-City University of New York (62413-0040) grants awarded to the author. The author gratefully acknowledges Marianna Ostrovskaya and Max Ruf for their assistance in data collection.

\section{References}

Berridge, K. C., Grill, H. J., \& Norgren, R. (1981). Relation of consummatory responses and preabsorptive insulin release to palatability and learned taste aversions. Journal of Comparative and Physiological Psychology, 95, 363-382.

Boakes, R. A. (2005). Persistence of acquired changes in the properties of odors and flavors for both humans and rats. Chemical Senses, 30(Suppl.1), i238-i239.

Bouton, M. E. (1991). Context and retrieval in extinction and in other examples of interference in simple associative learning. In L. Dachowski \& C. F. Flaherty (Eds.), Current topics in animal learning: Brain, emotion, and cognition (pp. 25-33). NJ: Hillsdale.

Bouton, M. E. (1993). Context, time, and memory retrieval in the interference paradigms of Pavlovian learning. Psychological Bulletin, 114, 80-99.

Bouton, M. E. (2004). Context and behavioral processes in extinction. Learning \& Memory, 11, 485-494.

Bouton, M. E., \& King, D. A. (1986). Effect of context on performance to conditioned stimuli with mixed histories of reinforcement and non reinforcement. Journal of Experimental Psychology. Animal Behavior Processes, 12, 4-15.

Bouton, M. E., \& Sunsay, C. (2003). Importance of trials versus accumulating time across trials in partially reinforced appetitive conditioning. Journal of Experimental Psychology. Animal Behavior Processes, 29, 62-77.

Capaldi, E. D., Myers, D. E., Campbell, D. H., \& Sheffer, J. D. (1983). Conditioned flavor preferences based on hunger level during original flavor exposure. Animal Learning \& Behavior, 11, 107-115.

De Houwer, J., Thomas, S., \& Baeyens, F. (2001). Associative learning of likes and dislikes: A review of 25 years of research on human evaluative conditioning. Psychological Bulletin, 127, 853-869.

De la Casa, L. G., Marquez, R., \& Lubow, R. E. (2009). Super-latent inhibition of conditioned taste preference with a long retention interval. Learning and Motivation, 40, 329-342.

Delamater, A. R. (1996). Effects of several extinction treatments upon the integrity of Pavlovian stimulus-outcome associations. Animal Learning \& Behavior, 24, 437-449.

Delamater, A. R. (2007). Extinction of conditioned flavor preferences. Journal of Experimental Psychology. Animal Behavior Processes, 33, 160-171.

Delamater, A. R., Campese, V., LoLordo, V. M., \& Sclafani, A. (2006). Unconditioned stimulus devaluation effects in nutrientconditioned flavor preferences. Journal of Experimental Psychology. Animal Behavior Processes, 32, 295-306.

Delamater, A. R., Campese, V., \& Westbrook, R. F. (2009). Renewal and spontaneous recovery, but not latent inhibition, are mediated by gamma-aminobutyric acid in appetitive conditioning. Journal of Experimental Psychology. Animal Behavior Processes, 35, 224-237.

Drucker, D. B., Ackroff, K., \& Sclafani, A. (1994). Nutrient-conditioned flavor preference and acceptance in rats: Effects of deprivation state and non reinforcement. Physiology \& Behavior, 56, 701-707.

Dwyer, D. M. (1999). Retrospective revaluation or mediated conditioning? The effect of different reinforcers. The Quarterly Journal of Experimental Psychology, 52B, 289-306.

Dwyer, D. M. (2005). Reinforcer devaluation in palatability-based learned flavor preferences. Journal of Experimental Psychology. Animal Behavior Processes, 31, 487-492.

Dwyer, D. M. (2008). Micro structural analysis of conditioned and unconditioned responses to maltodextrin. Learning \& Behavior, $36,149-157$.

Dwyer, D. M., Pincham, H. L., Thein, T., \& Harris, J. A. (2009). A learned flavor preference persists despite the extinction of conditioned hedonic reactions to the cue flavors. Learning \& Behavior, 37, 305-310.

Elizalde, G., \& Sclafani, A. (1990). Flavor preferences conditioned by intragastric polycose infusions: A detailed analysis using an electronic esophagus preparation. Physiology \& Behavior, 47, 63-77.

Gallistel, C. R., \& Gibbon, J. (2000). Time, rate, and conditioning. Psychological Review, 107, 289-344.

Gottlieb, D. A. (2005). Acquisition with partial and continuous reinforcement in rat magazine approach. Journal of Experimental Psychology. Animal Behavior Processes, 31, 319-333.

Halgren, C. R. (1974). Latent inhibition in rats: Associative or non associative? Journal of Comparative and Physiological Psychology, $86,74-78$

Harris, J. A., Shand, F. L., Carroll, L. Q., \& Westbrook, R. F. (2004). Persistence of preference for a flavor presented in simultaneous compound with sucrose. Journal of Experimental Psychology. Animal Behavior Processes, 30, 177-189.

Higgins, T., \& Rescorla, R. A. (2004). Extinction and retraining of simultaneous and successive flavor conditioning. Learning \& Behavior, 32, 213-219.

Leung, H. T., \& Westbrook, R. F. (2010). Increased spontaneous recovery with increases in conditioned stimulus alone exposures. 
Journal of Experimental Psychology: Animal Behavior Processes, 36, 354-367.

Lubow, R. E. (1989). Latent inhibition and conditioned attention theory. Cambridge: Cambridge University Press.

Pearce, J. M. (1987). A model for stimulus generalization in Pavlovian conditioning. Psychological Review, 94, 61-73.

Pearce, J. M. (1994). Similarity and discrimination: A selective review and a connectionist model. Psychological Review, 101, 587-607.

Pearce, J. M. (2002). Evaluation and development of a connectionist theory of configural learning. Animal Learning \& Behavior, 30, 73-95.

Pearce, J. M., \& Hall, G. (1980). A model for Pavlovian learning: Variations in the effectiveness of conditioned but not of unconditioned stimuli. Psychological Review, 87, 532-552.

Rescorla, R. A. (1971). Summation and retardation tests of latent inhibition. Journal of Comparative and Physiological Psychology, $75,77-81$.

Rescorla, R. A. (1996). Preservation of Pavlovian associations through extinction. The Quarterly Journal of Experimental Psychology, $49 B, 245-258$.

Rescorla, R. A. (1997). Spontaneous recovery after Pavlovian conditioning with multiple outcomes. Animal Learning \& Behavior, 25, 99-107.

Rescorla, R. A. (2004a). Spontaneous recovery. Learning \& Memory, $11,501-509$.

Rescorla, R. A. (2004b). Spontaneous recovery varies inversely with the training-extinction interval. Learning \& Behavior, 32, 401-408.

Rescorla, R. A. (2007). Spontaneous recovery after reversal and partial reinforcement. Learning \& Behavior, 35, 191-200.

Rescorla, R. A., \& Wagner, A. R. (1972). A theory of Pavlovian conditioning: Variations in the effectiveness of reinforcement and non reinforcement. In A. H. Black \& W. F. Prokasy (Eds.), Classical conditioning II: Current research and theory (pp. 64-99). New York: Appleton.

Rodger, R. S. (1974). Multiple contrasts, factors, error rate, and power. The British Journal of Mathematical and Statistical Psychology, 27, 179-198.

Rozeboom, W. W. (1958). "What is learned?" An empirical enigma. Psychological Review, 65, 22-33.

Scarlet, J., Campese, V., \& Delamater, A. R. (2009). Sensory-specific associations in flavor- preference reversal learning. Learning \& Behavior, 37, 179-187.

Sclafani, A., \& Nissenbaum, J. W. (1988). Robust conditioned flavor preference produced by intragastric starch infusions in rats. The American Journal of Physiology, 255, 672-675.

Stevenson, R. J., Boakes, R. A., \& Wilson, J. P. (2000). Resistance to extinction of conditioned odor perceptions: Evaluative conditioning is not unique. Journal of Experimental Psychology. Learning, Memory, and Cognition, 26, 423-440.

Tracy, A. L., \& Davidson, T. L. (2006). Comparison of nutritive and non nutritive stimuli in intestinal and oral conditioned taste aversion paradigms. Behavioral Neuroscience, 120, 1268-1278.

Tracy, A. L., Phillips, R. J., Chi, M. M., Powley, T. L., \& Davidson, T. L. (2004). The gastrointestinal tract "tastes" nutrients: Evidence from the intestinal taste aversion paradigm. American Journal of Physiology: Regulatory, Integrative and Comparative Physiology, 287, R1086-R1100.

Wagner, A. R. (1978). Expectancies and the priming of STM. In S. H. Hulse, H. Fowler, \& W. K. Honig (Eds.), Cognitive processes in animal behavior (pp. 177-209). NJ: Hillsdale.

Wagner, A. R. (2008). Evolution of an elemental theory of Pavlovian conditioning. Learning \& Behavior, 36, 253-265. 